\section{Management of multiple myeloma: the impact of ixazomib's approval in Canada}

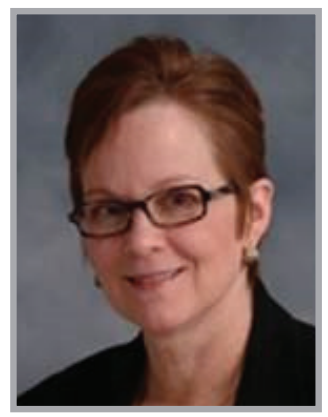

Donna E Reece* speaks to Laura Dormer, Commissioning Editor: Dr Donna E Reece is a Professor of Medicine and Director of the Program for Multiple Myeloma and Related Diseases in the Department of Medical Oncology and Hematology at Princess Margaret Hospital/University of Toronto. She earned a Bachelor of Arts degree at the University of Texas, Austin, and graduated as valedictorian with a medical degree from Baylor College of Medicine, Houston, Texas. She completed an internship in Internal Medicine at the University of Colorado Affiliated Hospitals, a residency and Chief Residency in Internal Medicine at Jewish Hospital, St Louis, and a Fellowship in Hematology/Oncology at Barnes Hospital, Washington University, St Louis, Missouri. She was a fellow and later a leukemia/stem cell transplant staff physician at Vancouver General Hospital/University of British Columbia for over 10 years. She then served as Director of the Outpatient Leukemia/Stem Cell Transplant Program, and later interim director, of the Blood and Marrow Transplant Program of the Markey Cancer Center at the University of Kentucky, Lexington, Kentucky until her appointment to Princess Margaret Hospital in Toronto in 2001. Dr Reece received the David and Molly Bloom Chair in Myeloma Research in 2009. She is currently the co-chair of the Multiple Myeloma Clinical Trials Group of the National Cancer Institute of Canada, member of the Scientific Advisory Board of the International Myeloma Foundation, and member of the Project Review Committee of the MMRC (Multiple Myeloma Research Consortium). She is also the Chief Medical Officer of the Myeloma Canada Research Network and serves on the board of directors of Myeloma Canada. Her career focus has been in the areas of hematopoietic stem cell transplantation, lymphoid malignancies and plasma cell dyscrasias. She has published numerous articles in these areas.

First draft submitted: 2 September 2016; Accepted for publication: 12 October 2016; Published online: 13 January 2017

Q Could you briefly summarize your career path \& how you came to work in your current role at Princess Margaret Hospital/University of Toronto?

I trained in the USA, at Baylor College of Medicine (TX, USA), then went on to complete a fellowship in hematology-oncology at Washington University in St Louis (MO, USA). For the past 30 years, I have primarily lived and worked in Canada, starting

*Department of Medical Oncology \& Hematology, Princess Margaret Cancer Centre, Toronto, ON, Canada donna.reece@uhn.ca

\section{KEYWORDS}

- NINLARO • personalized medicine

- refractory multiple myeloma

- relapsed multiple myeloma

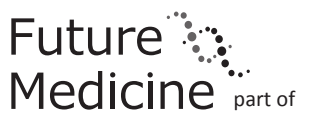


when I moved to Vancouver to help develop a bone marrow transplant program. At this time, bone marrow transplants began to emerge as a good treatment for myeloma, which piqued my interest in the disease. I worked in the bone marrow/stem cell transplantation field for 25 years, but really became involved in myeloma management when I moved to Princess Margaret Hospital/University of Toronto, which has a long legacy of myeloma research. Today, I have evolved into a full-time myeloma-focused doctor who still does stem cell transplants amid a number of other nontransplant therapies, including using exciting new drugs. It has not been the 'usual' path for a myeloma doctor, but has been a wonderful one. Autologous stem cell transplants continue to play an important role in the management of myeloma - a role that has not diminished over the last 20 years. Transplants were a key first step to improve disease management for myeloma patients historically, but there is now a pressing need for additional new drugs for use before and after relapse, and for any subsequent relapses.

\section{Q What is the current standard of care for} patients with relapsed \&/or refractory multiple myeloma?

I informally use the term 'early' relapse to refer to a myeloma patient's first or second relapse, and 'advanced' relapse when they have had recurrence three or more times. Most myeloma patients will receive three, four or even five lines of therapy before they unfortunately pass away from the disease. When explaining myeloma treatments to patients, I often refer to them as 'beads on a chain', with each treatment as a bead. We want each of those treatments to work as well as possible, for as long as possible and to be the least toxic. The 'beads' for 'early' relapse are different from those used for 'advanced' relapse. The disease becomes more stubborn - harder to treat and with shorter remission times - as subsequent relapses occur. There has been a tremendous amount of progress with options for first and second relapses. Often, particularly in the past, the standard-of-care in many jurisdictions has been the use of a two-drug regimen such as lenalidomide and dexamethasone in this setting. Other options are also available, including combinations of bortezomib, dexamethasone and perhaps another drug such as cyclophosphamide.
Q What are the main unmet needs for these patients?

The first need is to ensure that each line of therapy achieves the highest remission rate, the longest remission time and the best quality of life for patients. It is also important to be able to give a treatment for as long as possible before patients have to switch to the next line of treatment - because at this point they have lost a 'bead' in the chain.

Q How does NINLARO (ixazomib) differ from currently available treatments?

NINLARO has been approved by Health Canada for use in myeloma patients who have received one to three prior treatments. It has two important qualities; first, it is an oral treatment and is therefore the first oral drug in its class of proteasome inhibitors (PIs). This is clearly a tremendous advantage to patients, as although other PIs are effective treatments, traveling back and forth to the clinic is onerous. Many myeloma patients have bone disease and are in pain; transportation is also an issue, and many myeloma patients are elderly. Having an oral PI really meets a need for patients living with relapsed and refractory myeloma. Second, NINLARO appears to be effective in certain high-risk myeloma categories. Myeloma is not one disease, but is very heterogeneous; some patients experience a relatively indolent disease course, while others have an aggressive course - NINLARO can be used in all patients. It is approved to be given with lenalidomide and dexamethasone, so this is an approval for a three-drug regimen that is very effective.

\section{Q How will this affect the way patients in Canada are treated?}

NINLARO is a major step forward in the treatment of patient with relapsed myeloma. Importantly, it potentially makes PIs accessible to all patients - even those with long distances to travel to clinic. The fact that it is an oral treatment will improve quality of life for patients. It is also important to consider this from the perspective of the healthcare system. Canada has a single-payer system, and chemotherapy daycare units across the country are typically very full of patients requiring parenteral administration of their treatments. While there is a positive picture in terms of the emergence of many new myeloma drugs, the majority are given parenterally and the harder news is that chemotherapy daycare 
facilities remain limited. Hence, NINLARO also helps the healthcare system as it decreases utilization of these facilities.

Q What are the next steps for the research in this area?

The Holy Grail for myeloma is the development of personalized medicines. As mentioned, myeloma is a heterogeneous disease and therefore cannot have a one-size-fits-all treatment. In addition, going back to the 'beads on a chain' analogy, we always need new beads that are effective - and this means continuing the search for new classes of drugs for use in patients who become resistant to existing classes. Underpinning this is basic research, and it requires continued partnerships between the basic sciences, clinical researchers and patients themselves, to inform us on what is important to them. Patient-centered research is becoming increasingly important in the USA and elsewhere, and in this regard I believe we are ahead of the curve. Our Myeloma Canada Research Network partners with Myeloma Canada, the patients' group, and has patient involvement at every level of decision-making for clinical trials. Focus has to be on the patients, their experience and what is important to them. I believe our partnership with Myeloma Canada is unique in this regard, which is very exciting and very rewarding, and ensures the research is kept in focus and in keeping with patient priorities.

\section{Disclaimer}

The opinions expressed in this interview are those of the interviewee and do not necessarily reflect the views of Future Medicine Ltd.

Financial \& competing interests disclosure

DE Reece has received honoraria, consultancy and research funding from Takeda. DE Reece has no other relevant affiliations or financial involvement with any organization or entity with a financial interest in or financial conflict with the subject matter or materials discussed in the manuscript apart from those disclosed.

No writing assistance was utilized in the production of this manuscript. 